\title{
Wellposedness of the Inverse Problem for Dirac Operator
}

\author{
Murat Sat, ${ }^{1}$ Etibar S. Panakhov, ${ }^{2}$ and Kezban Tas ${ }^{3}$ \\ ${ }^{1}$ Department of Mathematics, Faculty of Science and Art, Erzincan University, 24100 Erzincan, Turkey \\ ${ }^{2}$ Department of Mathematics, Faculty of Science, Firat University, 23119 Elazig, Turkey \\ ${ }^{3}$ Tunceli University, 62000 Tunceli, Turkey \\ Correspondence should be addressed to Murat Sat; msat@erzincan.edu.tr
}

Received 29 July 2013; Accepted 10 September 2013

Academic Editors: Z.-C. Deng, Z. Shi, G. Toth, and Z. Wang

Copyright (c) 2013 Murat Sat et al. This is an open access article distributed under the Creative Commons Attribution License, which permits unrestricted use, distribution, and reproduction in any medium, provided the original work is properly cited.

We study the wellposedness of the inverse problem for Dirac operator. We consider two different problems (unperturbed and perturbed problem) for Dirac operator, and then we prove that if the spectral characteristics of these problems are close to each other, then the difference between their potential functions is sufficiently small.

\section{Introduction}

Inverse problems are studied for certain special classes of ordinary differential operators. Typically, in inverse eigenvalue problems, one measures the frequencies of a vibrating system and tries to infer some physical properties of the system. An early important result in this direction, which gave vital impetus for the further development of inverse problem theory, was obtained in [1-5].

The Dirac equation is a modern presentation of the relativistic quantum mechanics of electrons, intended to make new mathematical results accessible to a wider audience. It treats in some depth the relativistic invariance of a quantum theory, self-adjointness and spectral theory, qualitative features of relativistic bound and scattering states, and the external field problem in quantum electrodynamics, without neglecting the interpretational difficulties and limitations of the theory.

Inverse problems for Dirac system had been investigated by Moses [6], Prats and Toll [7], Verde [8], Gasymov and Levitan [9], and Panakhov [10, 11]. It is well known [12] that two spectra uniquely determine the matrix-valued potential function. In particular, in [13], eigenfunction expansions for one-dimensional Dirac operators describing the motion of a particle in quantum mechanics are investigated. Recently, Dirac operators have been extensively studied [14-19].

Mizutani showed the wellposedness problem of the Sturm-Liouville operator according to norming constants and eigenvalues [20]. The purpose of this paper is to give the wellposedness problem for Dirac operator by using Mizutani's method.

Let $L$ denote a matrix operator

$$
L \equiv\left(\begin{array}{ll}
p_{11}(x) & p_{12}(x) \\
p_{21}(x) & p_{22}(x)
\end{array}\right), \quad p_{12}(x)=p_{21}(x),
$$

where the $p_{i k}(x)(i, k=1,2)$ are real functions which are defined and continuous on the interval $[0, \pi]$. Further, let $\varphi(x, \lambda)$ denote a two-component vector-function

$$
\varphi(x, \lambda)=\left(\begin{array}{l}
\varphi_{1}(x, \lambda) \\
\varphi_{2}(x, \lambda)
\end{array}\right) .
$$

Then the equation

$$
\left(B \frac{d}{d x}+L-\lambda I\right) \varphi=0,
$$

where $\lambda$ is a parameter and

$$
B \equiv\left(\begin{array}{cc}
0 & 1 \\
-1 & 0
\end{array}\right), \quad I \equiv\left(\begin{array}{ll}
1 & 0 \\
0 & 1
\end{array}\right),
$$

is equivalent to the system of two simultaneous first-order ordinary differential equations

$$
\begin{aligned}
\frac{d \varphi_{2}}{d x}+p_{11}(x) \varphi_{1}+p_{12}(x) \varphi_{2} & =\lambda \varphi_{1}, \\
-\frac{d \varphi_{1}}{d x}+p_{21}(x) \varphi_{1}+p_{22}(x) \varphi_{2} & =\lambda \varphi_{2} .
\end{aligned}
$$


For the case in which $p_{12}(x)=p_{21}(x)=0, p_{11}(x)=$ $V(x)+m, p_{22}(x)=V(x)-m$, where $V(x)$ is a potential and $m$ is the mass of a particle, the system (5) is known in relativistic quantum theory as a stationary one-dimensional Dirac system [5].

Consider the following eigenvalue problem for Dirac operator:

$$
\begin{gathered}
B y^{\prime}+Q(x) y=\lambda y, \quad 0 \leq x \leq \pi, \\
y_{1}(0) \cos \alpha+y_{2}(0) \sin \alpha=0, \\
y_{1}(\pi)=0,
\end{gathered}
$$

where $Q(x)=\left(\begin{array}{cc}p(x) & q(x) \\ q(x) & -p(x)\end{array}\right), y(x)=\left(\begin{array}{l}y_{1}(x) \\ y_{2}(x)\end{array}\right), p(x)$ and $q(x) \epsilon$ $C^{1}[0, \pi]$ are real-valued functions, $0 \leq \alpha \leq \pi$, and $\lambda$ is spectral parameter.

We denote by

$$
\varphi(x, \lambda)=\left(\begin{array}{c}
\varphi_{1}(x, \lambda) \\
\varphi_{2}(x, \lambda)
\end{array}\right)=\left(\begin{array}{c}
\sin (\lambda x-\alpha)+O\left(\frac{e^{\tau x}}{\lambda}\right) \\
\cos (\lambda x-\alpha)+O\left(\frac{e^{\tau x}}{\lambda}\right)
\end{array}\right)
$$

the solution of (6) satisfying the initial conditions

$$
\varphi_{1}(0, \lambda)=-\sin \alpha, \quad \varphi_{2}(0, \lambda)=\cos \alpha,
$$

where $\tau=|\operatorname{Im} \lambda|$.

We show the spectral characteristics of the problems (6)(8) by $\left\{\lambda_{n}, \rho_{n}\right\}(n \in \mathbb{Z})$, where $\left\{\lambda_{n}\right\}$ is the spectrum (set of eigenvalues) and $\left\{\rho_{n}\right\}$ is the norming constants of this problem. These spectral characteristics satisfy the following asymptotic expressions, respectively [9]:

$$
\begin{gathered}
\lambda_{n}=n-\frac{\alpha}{\pi}+\frac{\alpha_{1}}{n}+O\left(\frac{1}{n^{2}}\right), \\
\rho_{n}=\pi+\frac{c_{1}}{n}+O\left(\frac{1}{n^{2}}\right),
\end{gathered}
$$

where $\alpha, \alpha_{1}$, and $c_{1}$ are real numbers.

Now let us consider the second eigenvalue problem

$$
B y^{\prime}+\widetilde{Q}(x) y=\mu y, \quad 0 \leq x \leq \pi,
$$

with the same boundary conditions (7), (8), where $\widetilde{Q}(x)=$ $\left(\begin{array}{cc}\tilde{p}(x) & \widetilde{q}(x) \\ \widetilde{q}(x) & -\widetilde{p}(x)\end{array}\right), \tilde{p}(x)$ and $\tilde{q}(x) \in C^{1}[0, \pi]$ are real-valued functions and $\mu$ is spectral parameter. We denote the spectral characteristics of the problems (12), (7), and (8) by $\left\{\mu_{n}, \sigma_{n}\right\}(n \in \mathbb{Z})$, where $\left\{\mu_{n}\right\}$ is the spectrum and $\left\{\sigma_{n}\right\}$ is the norming constants of this problem. These spectral characteristics satisfy the following asymptotic expressions, respectively:

$$
\begin{gathered}
\mu_{n}=n-\frac{\alpha^{\prime}}{\pi}+\frac{\alpha_{1}^{\prime}}{n}+O\left(\frac{1}{n^{2}}\right), \\
\sigma_{n}=\pi+\frac{c_{1}^{\prime}}{n}+O\left(\frac{1}{n^{2}}\right),
\end{gathered}
$$

where $\alpha^{\prime}, \alpha_{1}^{\prime}$, and $c_{1}^{\prime}$ are real constants.
Theorem 1. Let $E$ be a linear topological space and $E_{1}, E_{2} \subset$ $E$, where $E_{1}$ and $E_{2}$ are linear topological subspaces of $E$. The transformation operator $X=X_{A, B}$ mapping $E_{1}$ to $E_{2}$ can be defined as follows [9]:

$$
X[\varphi(x, \lambda)]=\widetilde{\varphi}(x, \lambda)=\varphi(x, \lambda)+\int_{0}^{x} K(x, s) \varphi(s, \lambda) d s
$$

where the kernel $K(x, s)$ is a solution of the equation

$$
B \frac{\partial K}{\partial x}+\widetilde{Q}(x) K(x, s)=-\frac{\partial K}{\partial s} B+Q(x) K(x, s)
$$

and also satisfies the following conditions:

$$
\begin{gathered}
B K(x, x)-K(x, x) B=\widetilde{Q}(x)-Q(x), \\
K_{21}(x, 0)=K_{11}(x, 0)=0,
\end{gathered}
$$

and $K(x, s)=\left(\begin{array}{l}K_{11}(x, s) K_{12}(x, s) \\ K_{21}(x, s) K_{22}(x, s)\end{array}\right)$ is continuously differentiable on $[0, \pi]$.

The $K(x, s)$ function in (14) satisfies the following integral equation:

$$
\begin{array}{r}
K(x, s)+F(x, s)+\int_{0}^{x} K(x, t) F(t, s) d t=0 \\
0 \leq s \leq x \leq \pi
\end{array}
$$

where $F(x, s)$ function is as such

$$
F(x, s)=\sum_{n=1}^{\infty} \frac{\varphi\left(x, \mu_{n}\right) \varphi^{T}\left(s, \mu_{n}\right)}{\sigma_{n}}-\frac{\varphi\left(x, \lambda_{n}\right) \varphi^{T}\left(s, \lambda_{n}\right)}{\rho_{n}}
$$

and $F(x, s)=\left(\begin{array}{ll}F_{11}(x, s) & F_{12}(x, s) \\ F_{21}(x, s) & F_{22}(x, s)\end{array}\right)$ is continuously differentiable on $[0, \pi]$.

Now we present main theorem in this paper.

\section{Main Results}

Theorem 2. If

$$
M \equiv \sum_{n=1}^{\infty}\left\{\left|\sigma_{n}-\alpha_{n}\right|+\left|\mu_{n}-\lambda_{n}\right|\right\}
$$

is sufficiently small, then we have

$$
\begin{aligned}
& \max _{0 \leq x \leq \pi}|\tilde{p}(x)-p(x)| \leq C^{\prime} M, \\
& \max _{0 \leq x \leq \pi}|\widetilde{q}(x)-q(x)| \leq C^{\prime \prime} M,
\end{aligned}
$$

where $C^{\prime}>0$ and $C^{\prime \prime}>0$ are constants. 
Proof. Let us solve the Gelfand-Levitan equation (18). We start from $F(s, t)$ and construct the iterated kernels $F^{(n)}(s, t ; x)(n=1,2,3, \ldots)$ as follows:

$$
\begin{gathered}
F^{(1)}(s, t ; x)=F(s, t), \\
F^{(n+1)}(s, t ; x)=\int_{0}^{x} F(s, u) F^{(n)}(u, t ; x) d u,
\end{gathered}
$$

for $n \geq 1$. that

We put $S(s, t ; x)=\sum_{n=1}^{\infty}(-1)^{n} F^{(n)}(s, t ; x)$ and assume

$$
\iint_{0}^{\pi}|F(s, t)|^{2} d s d t<1
$$

Then it is easily seen that

$$
K(x, s)=S(x, s ; x), \quad \text { for } 0 \leq s \leq x \leq \pi .
$$

From (19), we have

$$
\begin{gathered}
F(x, s)=\sum_{n=1}^{\infty}\left[\frac{\varphi\left(x, \mu_{n}\right) \varphi^{T}\left(s, \mu_{n}\right)}{\sigma_{n}}-\frac{\varphi\left(x, \lambda_{n}\right) \varphi^{T}\left(s, \lambda_{n}\right)}{\rho_{n}}\right] \\
=\sum_{n=1}^{\infty}\left(\frac{1}{\sigma_{n}}\left[\begin{array}{l}
\left.\varphi_{1}\left(x, \mu_{n}\right)\right] \\
\varphi_{2}\left(x, \mu_{n}\right)
\end{array}\right]\left[\varphi_{1}\left(s, \mu_{n}\right) \varphi_{2}\left(s, \mu_{n}\right)\right]\right. \\
\left.\left.-\frac{1}{\rho_{n}}\left[\begin{array}{l}
\left.\varphi_{1}\left(x, \lambda_{n}\right)\right] \\
\varphi_{2}\left(x, \lambda_{n}\right)
\end{array}\right] \varphi_{1}\left(s, \lambda_{n}\right) \varphi_{2}\left(s, \lambda_{n}\right)\right]\right) \\
=\sum_{n=1}^{\infty}\left\{\left[\begin{array}{c}
\frac{\varphi_{1}\left(x, \mu_{n}\right) \varphi_{1}\left(s, \mu_{n}\right)}{\sigma_{n}} \\
-\frac{\varphi_{1}\left(x, \lambda_{n}\right) \varphi_{1}\left(s, \lambda_{n}\right)}{\rho_{n}} \\
\times \frac{\varphi_{1}\left(x, \mu_{n}\right) \varphi_{2}\left(s, \mu_{n}\right)}{\sigma_{n}} \\
-\frac{\varphi_{1}\left(x, \lambda_{n}\right) \varphi_{2}\left(s, \lambda_{n}\right)}{\rho_{n}} \\
\frac{\varphi_{2}\left(x, \mu_{n}\right) \varphi_{1}\left(s, \mu_{n}\right)}{\sigma_{n}} \\
-\frac{\varphi_{2}\left(x, \lambda_{n}\right) \varphi_{1}\left(s, \lambda_{n}\right)}{\rho_{n}} \\
-\frac{\varphi_{2}\left(x, \lambda_{n}\right) \varphi_{2}\left(s, \lambda_{n}\right)}{\rho_{n}} \\
\times \frac{\varphi_{2}\left(x, \mu_{n}\right) \varphi_{2}\left(s, \mu_{n}\right)}{\sigma_{n}}
\end{array}\right]\right.
\end{gathered}
$$

From (25), it is easy to see that

$$
\begin{aligned}
& F_{11}(x, s)=\sum_{n=1}^{\infty}\left[\frac{\varphi_{1}\left(x, \mu_{n}\right) \varphi_{1}\left(s, \mu_{n}\right)}{\sigma_{n}}-\frac{\varphi_{1}\left(x, \lambda_{n}\right) \varphi_{1}\left(s, \lambda_{n}\right)}{\rho_{n}}\right], \\
& F_{12}(x, s)=\sum_{n=1}^{\infty}\left[\frac{\varphi_{1}\left(x, \mu_{n}\right) \varphi_{2}\left(s, \mu_{n}\right)}{\sigma_{n}}-\frac{\varphi_{1}\left(x, \lambda_{n}\right) \varphi_{2}\left(s, \lambda_{n}\right)}{\rho_{n}}\right],
\end{aligned}
$$

$$
F_{21}(x, s)=\sum_{n=1}^{\infty}\left[\frac{\varphi_{2}\left(x, \mu_{n}\right) \varphi_{1}\left(s, \mu_{n}\right)}{\sigma_{n}}-\frac{\varphi_{2}\left(x, \lambda_{n}\right) \varphi_{1}\left(s, \lambda_{n}\right)}{\rho_{n}}\right],
$$

$$
F_{22}(x, s)=\sum_{n=1}^{\infty}\left[\frac{\varphi_{2}\left(x, \mu_{n}\right) \varphi_{2}\left(s, \mu_{n}\right)}{\sigma_{n}}-\frac{\varphi_{2}\left(x, \lambda_{n}\right) \varphi_{2}\left(s, \lambda_{n}\right)}{\rho_{n}}\right] .
$$

Firstly, we calculate formula of (26). Adding and subtracting $\varphi_{1}\left(x, \lambda_{n}\right) \varphi_{1}\left(s, \lambda_{n}\right) / \sigma_{n}$ to the right side of (26), then we obtain

$$
\begin{aligned}
F_{11}(x, s)= & \sum_{n=1}^{\infty}\left[\frac{\varphi_{1}\left(x, \mu_{n}\right) \varphi_{1}\left(s, \mu_{n}\right)}{\sigma_{n}}-\frac{\varphi_{1}\left(x, \lambda_{n}\right) \varphi_{1}\left(s, \lambda_{n}\right)}{\rho_{n}}\right] \\
& +\sum_{n=1}^{\infty}\left[\frac{\varphi_{1}\left(x, \lambda_{n}\right) \varphi_{1}\left(s, \lambda_{n}\right)}{\sigma_{n}}\right. \\
& \left.-\frac{\varphi_{1}\left(x, \lambda_{n}\right) \varphi_{1}\left(s, \lambda_{n}\right)}{\sigma_{n}}\right] \\
= & \sum_{n=1}^{\infty}\left[\left(\frac{\rho_{n}-\sigma_{n}}{\rho_{n} \sigma_{n}}\right) \varphi_{1}\left(x, \lambda_{n}\right) \varphi_{1}\left(s, \lambda_{n}\right)\right] \\
& +\frac{1}{\sigma_{n}} \int_{\lambda_{n}}^{\mu_{n}}\left[\varphi_{1}(x, \lambda) \varphi_{1}(s, \lambda)\right] d \lambda
\end{aligned}
$$

where $=\partial / \partial \lambda$. If the absolute value is taken in the formula of (30) and (9) is used and $s=x$ is written, the following is obtained:

$$
\begin{aligned}
\left|F_{11}(x, x)\right| & \leq \sum_{n=1}^{\infty}\left[\left|\left(\frac{\rho_{n}-\sigma_{n}}{\rho_{n} \sigma_{n}}\right) c_{1}\right|+\left|\frac{1}{\sigma_{n}} \int_{\lambda_{n}}^{\mu_{n}} c_{2} d \lambda\right|\right] \\
& \leq \sum_{n=1}^{\infty}\left[\left|\left(\rho_{n}-\sigma_{n}\right) c_{3}\right|+\left|c_{4} \int_{\lambda_{n}}^{\mu_{n}} d \lambda\right|\right] \\
& \leq C_{1} \sum_{n=1}^{\infty}\left\{\left[\left|\sigma_{n}-\rho_{n}\right|+\left|\mu_{n}-\lambda_{n}\right|\right]\right\}
\end{aligned}
$$

where $c_{1}, c_{2}, c_{3}, c_{4}$, and $C_{1}$ are constants and positive numbers. From (20), we have

$$
\left|F_{11}(x, x)\right| \leq C_{1} M
$$


From (24) and (32) for $n=1$, we have

$$
\begin{gathered}
F_{11}^{(2)}(s, t ; x)=\int_{0}^{x} F_{11}(s, u) F_{11}^{(1)}(u, t ; x) d u \\
\left|F_{11}^{(2)}(s, t ; x)\right| \leq \frac{\left(C_{1} M \pi\right)^{2}}{\pi}
\end{gathered}
$$

similarly for $n=2$

$$
\begin{aligned}
F_{11}^{(3)}(s, t ; x) & =\int_{0}^{x} F_{11}(s, u) F_{11}^{(2)}(u, t ; x) d u \\
\left|F_{11}^{(3)}(s, t ; x)\right| & \leq \frac{\left(C_{1} M \pi\right)^{3}}{\pi},
\end{aligned}
$$

consequently,

$$
\begin{gathered}
F_{11}^{(n)}(s, t ; x)=\int_{0}^{x} F_{11}(s, u) F_{11}^{(n-1)}(u, t ; x) d u \\
\left|F_{11}^{(n)}(s, t ; x)\right| \leq \frac{\left(C_{1} M \pi\right)^{n}}{\pi} .
\end{gathered}
$$

From (24), we get the following:

$$
K_{11}(x, s) \leq \sum_{n=1}^{\infty}(-1)^{n} F_{11}^{(n)}(s, t ; x) .
$$

From (35) and (36), we have

$$
\left|K_{11}(x, x)\right| \leq \sum_{n=1}^{\infty}\left|(-1)^{n} \frac{\left(C_{1} M \pi\right)^{n}}{\pi}\right|=\sum_{n=1}^{\infty} \frac{\left(C_{1} M \pi\right)^{n}}{\pi} .
$$

If $C_{1} M \pi$ is small, for example, $C_{1} M \pi<1 / 2$, then we have

$$
\begin{aligned}
\left|K_{11}(x, x)\right| & \leq \sum_{n=1}^{\infty} \frac{\left(C_{1} M \pi\right)^{n}}{\pi} \\
& =C_{1} M+\pi\left(C_{1} M\right)^{2}+\pi^{2}\left(C_{1} M\right)^{3}+\cdots \\
& \leq C_{1} M\left(1+\frac{1}{2}+\left(\frac{1}{2}\right)^{2}+\cdots\right) \\
& \leq 2 C_{1} M .
\end{aligned}
$$

If the operations for $F_{11}(x, s)$ are repeated, we get the following:

$$
\begin{aligned}
\left|F_{12}(x, s)\right| & \leq \sum_{n=1}^{\infty}\left[\left|\left(\frac{\rho_{n}-\sigma_{n}}{\rho_{n} \sigma_{n}}\right) c_{5}\right|+\left|\frac{1}{\sigma_{n}} \int_{\lambda_{n}}^{\mu_{n}} c_{6} d \lambda\right|\right], \\
& \leq \sum_{n=1}^{\infty}\left[\left|\left(\rho_{n}-\sigma_{n}\right) c_{7}\right|+\left|c_{8} \int_{\lambda_{n}}^{\mu_{n}} d \lambda\right|\right], \\
& \leq C_{2} \sum_{n=1}^{\infty}\left[\left|\sigma_{n}-\rho_{n}\right|+\left|\mu_{n}-\lambda_{n}\right|\right]
\end{aligned}
$$

where $c_{5}, c_{6}, c_{7}, c_{8}$, and $C_{2}$ are constants and positive numbers.
From (20), we have

$$
\left|F_{12}(x, s)\right| \leq C_{2} M .
$$

Similarly from (24) and (40) for $n=1$, we have

$$
\begin{gathered}
F_{12}^{(2)}(s, t ; x)=\int_{0}^{x} F_{12}(s, u) F_{12}^{(1)}(u, t ; x) d u \\
\left|F_{12}^{(2)}(s, t ; x)\right| \leq \frac{\left(C_{2} M \pi\right)^{2}}{\pi},
\end{gathered}
$$

for $n=2$,

$$
\begin{gathered}
F_{12}^{(3)}(s, t ; x)=\int_{0}^{x} F_{12}(s, u) F_{12}^{(2)}(u, t ; x) d u \\
\left|F_{12}^{(3)}(s, t ; x)\right| \leq \frac{\left(C_{2} M \pi\right)^{3}}{\pi},
\end{gathered}
$$

consequently,

$$
\begin{aligned}
F_{12}^{(n)}(s, t ; x) & =\int_{0}^{x} F_{12}(s, u) F_{12}^{(n-1)}(u, t ; x) d u, \\
\left|F_{12}^{(n)}(s, t ; x)\right| & \leq \frac{\left(C_{2} M \pi\right)^{n}}{\pi} .
\end{aligned}
$$

From (24), we get the following:

$$
K_{12}(x, s)=\sum_{n=1}^{\infty}(-1)^{n} F_{12}^{(n)}(s, t ; x) .
$$

If we repeat the above operations, we have

$$
\left|K_{12}(x, x)\right| \leq \sum_{n=1}^{\infty}\left|(-1)^{n} \frac{\left(C_{2} M \pi\right)^{n}}{\pi}\right|=\sum_{n=1}^{\infty} \frac{\left(C_{2} M \pi\right)^{n}}{\pi} .
$$

If $C_{2} M \pi$ is small, for example, $C_{2} M \pi<1 / 2$, then we have

$$
\begin{aligned}
\left|K_{12}(x, x)\right| & \leq \sum_{n=1}^{\infty} \frac{\left(C_{2} M \pi\right)^{n}}{\pi} \\
& =C_{2} M+\pi\left(C_{2} M\right)^{2}+\pi^{2}\left(C_{2} M\right)^{3}+\cdots \\
& \leq C_{2} M\left(1+\frac{1}{2}+\left(\frac{1}{2}\right)^{2}+\cdots\right) \\
& \leq 2 C_{2} M
\end{aligned}
$$

When the similar operations are repeated, the following is obtained:

$$
\begin{aligned}
& \left|K_{21}(x, x)\right| \leq 2 C_{3} M, \\
& \left|K_{22}(x, x)\right| \leq 2 C_{4} M,
\end{aligned}
$$

where $C_{3}>0$ and $C_{4}>0$ are constants. 
On the other side, from (16) we get the following:

$$
\begin{aligned}
B K(x, x)-K(x, x) B= & \left(\begin{array}{cc}
0 & 1 \\
-1 & 0
\end{array}\right)\left(\begin{array}{ll}
K_{11} & K_{12} \\
K_{21} & K_{22}
\end{array}\right) \\
& -\left(\begin{array}{ll}
K_{11} & K_{12} \\
K_{21} & K_{22}
\end{array}\right)\left(\begin{array}{cc}
0 & 1 \\
-1 & 0
\end{array}\right) \\
= & \left(\begin{array}{cc}
K_{12}+K_{21} & K_{22}-K_{11} \\
K_{22}-K_{11} & -K_{12}-K_{21}
\end{array}\right) \\
= & \left(\begin{array}{ll}
K_{12}+K_{21} & K_{22}-K_{11} \\
K_{22}-K_{11} & -K_{12}-K_{21}
\end{array}\right) \\
= & \left(\begin{array}{ll}
\widetilde{p}(x)-p(x) & \widetilde{q}(x)-q(x) \\
\widetilde{q}(x)-q(x) & p(x)-\widetilde{p}(x)
\end{array}\right) .
\end{aligned}
$$

From here, we have

$$
\begin{aligned}
& \tilde{p}(x)-p(x)=K_{12}+K_{21}, \\
& \widetilde{q}(x)-q(x)=K_{22}-K_{11} .
\end{aligned}
$$

Consequently from (38), (46), (47), and (49), we obtained the desired estimate

$$
\begin{aligned}
& \max _{0 \leq x \leq \pi}|\tilde{p}(x)-p(x)| \leq C^{\prime} M, \\
& \max _{0 \leq x \leq \pi}|\tilde{q}(x)-q(x)| \leq C^{\prime \prime} M,
\end{aligned}
$$

where $C^{\prime}>0$ and $C^{\prime \prime}>0$ are constants.

\section{Conclusion}

The more norming constants and spectrums which are taken as spectral characteristics of Dirac operators are close to each other, the more difference of potential functions is sufficiently small.

\section{References}

[1] V. Ambarzumian, "Über eine frage der eigenwerttheorie," Zeitschrift für Physik, vol. 53, no. 9-10, pp. 690-695, 1929.

[2] G. Borg, "Eine umkehrung der Sturm-Liouvilleschen eigenwertaufgabe," Acta Mathematica, vol. 78, no. 1, pp. 1-96, 1946.

[3] N. Levinson, “The inverse Sturm-Liouville problem," Matematisk Tidsskrift B, pp. 25-30, 1949.

[4] I. M. Gelfand and B. M. Levitan, "On the determination of a diderential equation from its spectral function," American Mathematical Society Translations, vol. 1, pp. 253-304, 1951.

[5] B. M. Levitan and I. S. Sargsjan, Introduction to Spectral Theory, American Mathematical Society, Providence, RI, USA, 1975.

[6] H. E. Moses, "Calculation of the scattering potentials from reflection coefficient," Bulletin of the American Physical Society, vol. 4, p. 240, 1956.

[7] F. Prats and J. S. Toll, "Construction of the Dirac equation central potential from phase shifts and bound states," Physical Review, vol. 113, no. 1, pp. 363-370, 1959.

[8] M. Verde, "The inversion problem in wave mechanics and dispersion relations," Nuclear Physics, vol. 9, no. 2, pp. 255-266, 1958.
[9] M. G. Gasymov and B. M. Levitan, "The inverse problem for a Dirac system,” Doklady Akademii Nauk SSSR, vol. 167, pp. 967970, 1966.

[10] E. S. Panakhov, "The defining of Dirac system in two incompletely set collection of eigenvalues," Doklady Akademii Nauk USSR, vol. 5, pp. 8-12, 1985.

[11] E. S. Panakhov, "Inverse problem for Dirac system in two partially settled spectrum," VINITY, vol. 3304, pp. 1-29, 1981.

[12] M. G. Gasymov and T. T. Dzhabiev, Determination of a system of Dirac diaerential equations using two spectra, Transactions of the summer school on spectral theory of operators, Baku, Azerbaijan, pp. 46-71, 1975.

[13] I. Joó and A. Minkin, "Eigenfunction estimate for a Dirac operator," Acta Mathematica Hungarica, vol. 76, no. 4, pp. 337349, 1997.

[14] B. Keskin and A. S. Ozkan, "Inverse spectral problems for Dirac operator with eigenvalue dependent boundary and jump conditions," Acta Mathematica Hungarica, vol. 130, no. 4, pp. 309-320, 2011.

[15] C. F. Yang, "Hochstadt-Lieberman theorem for Dirac operator with eigenparameter dependent boundary conditions," Nonlinear Analysis: Theory, Methods and Applications, vol. 74, no. 7, pp. 2475-2484, 2011.

[16] E. S. Panakhov, E. Yilmaz, and H. Koyunbakan, "Inverse nodal problem for Dirac operator," World Applied Sciences Journal, vol. 11, pp. 906-911, 2010.

[17] N. B. Kerimov, "A boundary value problem for the Dirac system with a spectral parameter in the boundary conditions," Differential Equations, vol. 38, no. 2, pp. 164-174, 2002.

[18] I. M. Nabiev, "On reconstruction of Dirac operator on the segment," Proceeding of IMM of Nas of Azerbaijan, vol. 18, pp. 97-102, 2003.

[19] M. S. Agranovich, "Spectral problems for the Dirac system with spectral parameter in local boundary conditions," Functional Analysis and Its Applications, vol. 35, no. 3, pp. 161-175, 2001.

[20] A. Mizutani, “On the inverse Sturm-Liouville problem," Journal of the Faculty of Science, The University of Tokyo IA, vol. 31, pp. 319-350, 1984. 


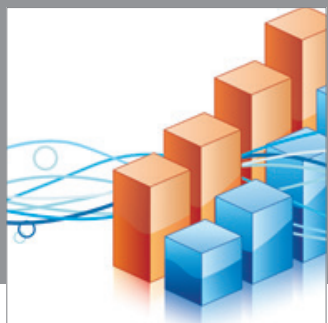

Advances in

Operations Research

mansans

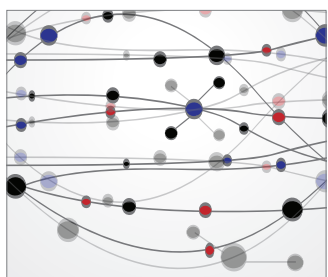

The Scientific World Journal
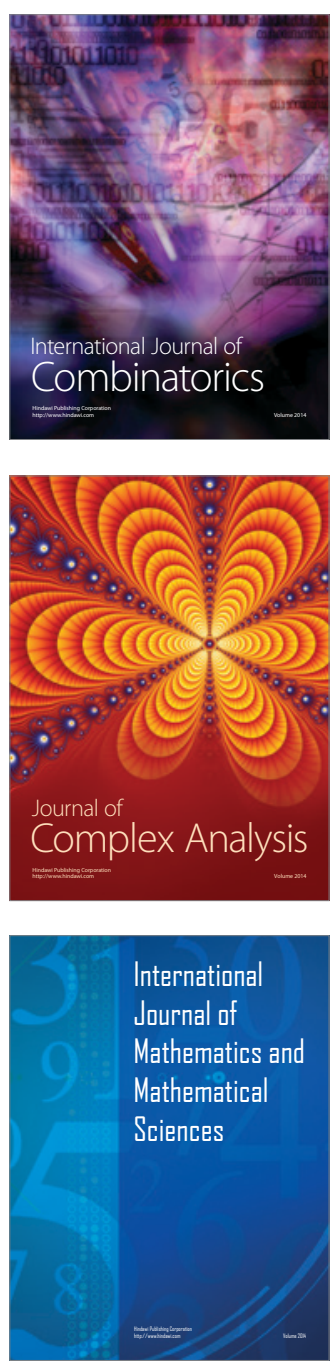
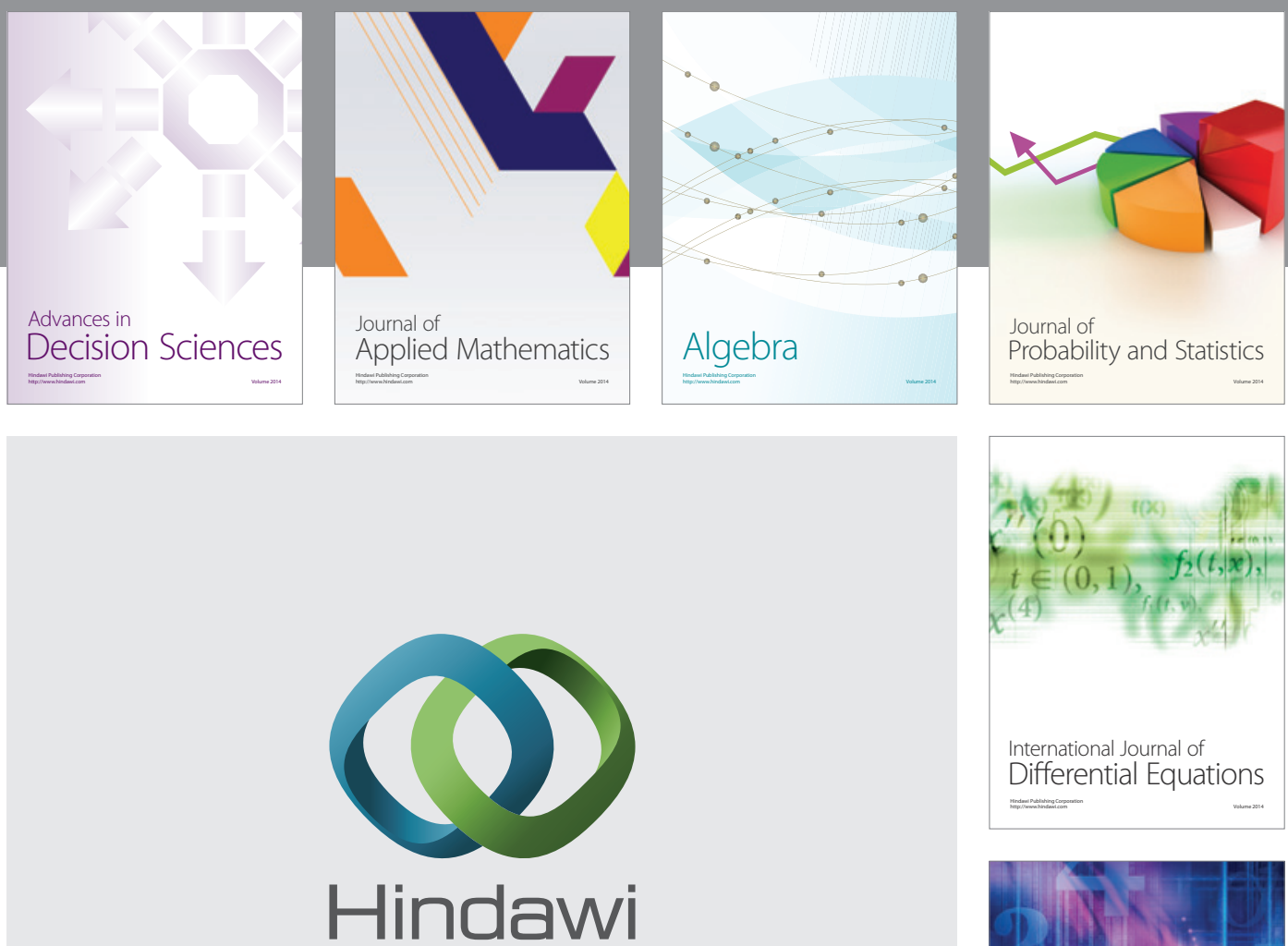

Submit your manuscripts at http://www.hindawi.com
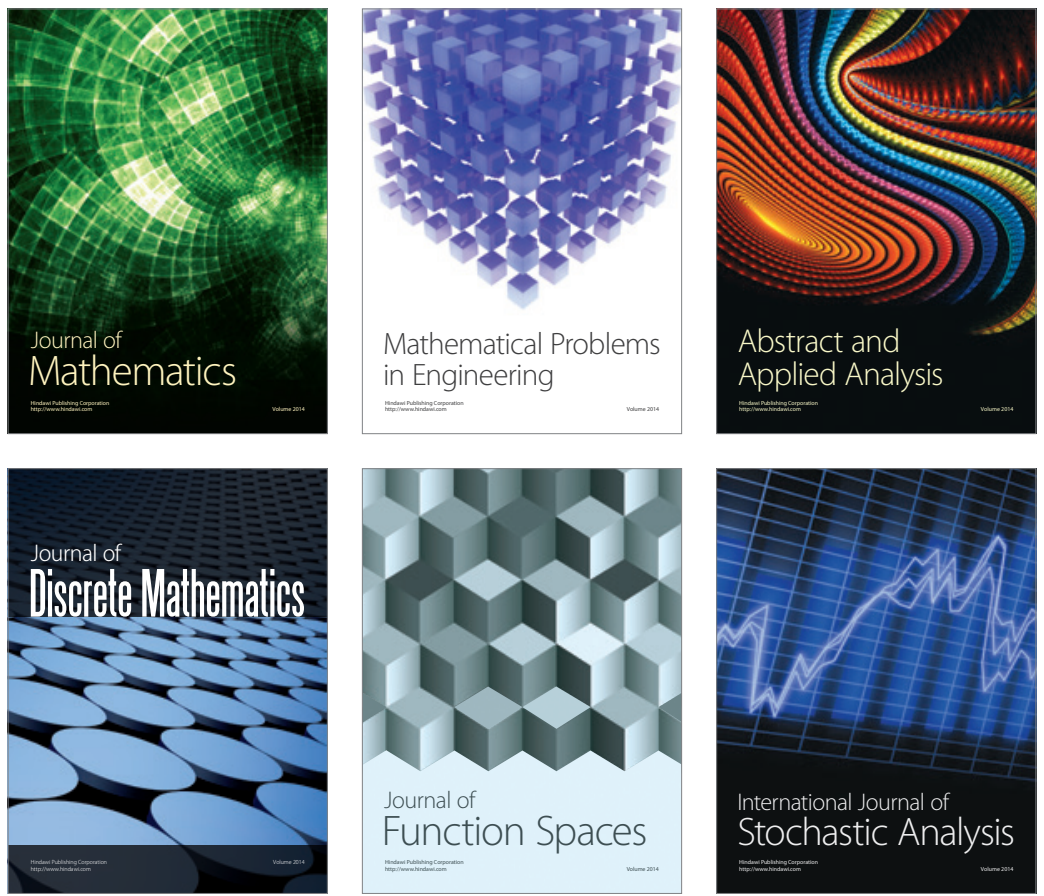

Journal of

Function Spaces

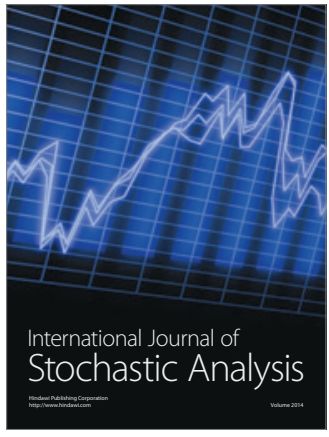

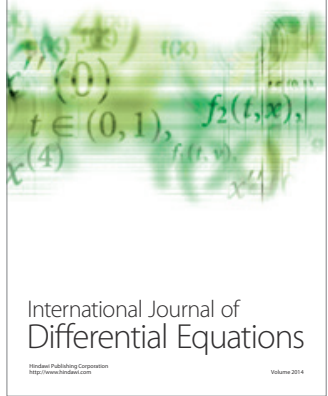
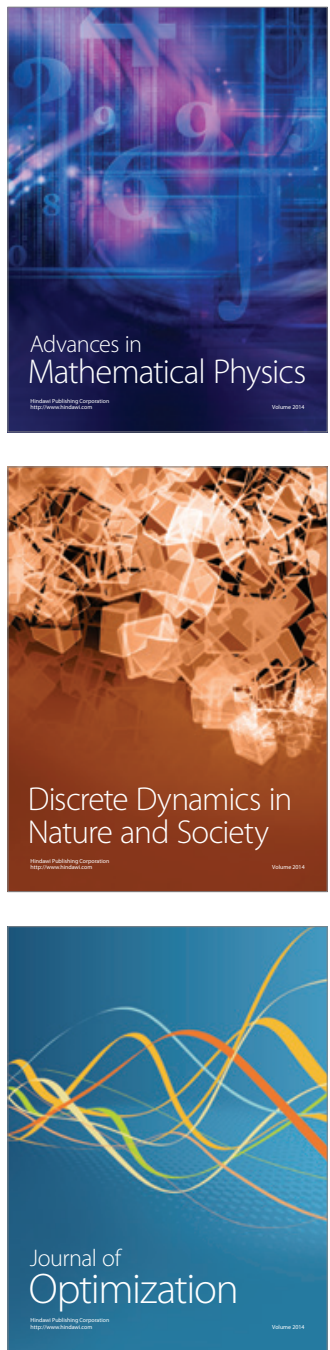